\title{
L'éducation relative à l'environnement en enseignement des sciences et de la technologie : une contribution pour mieux Vivre ensemble sur Terre
}

\section{Environmental education in science and technology classes} contribute to better "sharing the planet"

\section{La educación relativa al entorno ecológico en la enseñanza de ciencias y tecnología: una contribución para mejor «Vivir juntos sobre la Tierra»}

\author{
Patrick Charland, Patrice Potvin et Martin Riopel
}

Volume 37, numéro 2, automne 2009

Vivre ensemble, sur Terre

URI : https://id.erudit.org/iderudit/038816ar

DOI : https://doi.org/10.7202/038816ar

Aller au sommaire du numéro

Éditeur(s)

Association canadienne d'éducation de langue française

ISSN

0849-1089 (imprimé)

1916-8659 (numérique)

Découvrir la revue

Citer cet article

Charland, P., Potvin, P. \& Riopel, M. (2009). L'éducation relative à l'environnement en enseignement des sciences et de la technologie : une contribution pour mieux Vivre ensemble sur Terre. Éducation et francophonie, 37(2), 63-78. https://doi.org/10.7202/038816ar
Résumé de l'article

Dans les années soixante, les sociétés du monde entier ont graduellement pris conscience de l'ampleur, de la sévérité et de la complexité des divers problèmes environnementaux qui touchent notre planète. À travers de multiples sommets, congrès et colloques, qui ont donné lieu à de nombreuses déclarations, chartes ou stratégies, le champ de l'éducation relative à l'environnement s'est graduellement constitué. Malgré des intentions et des engagements fermes exprimés par les décideurs politiques et curriculaires, il semble que les initiatives en éducation relative à l'environnement (ERE) se soient plus développées dans les milieux éducatifs non formels ou en contexte informel qu'en milieu scolaire. On constate cependant une tendance récente à l'intégration d'éléments d'éducation relative à l'environnement dans les curriculums, et particulièrement dans les programmes de sciences du Québec. C'est donc dans cette perspective de l'intégration de l'ERE au sein des programmes de sciences que sera traitée la question du Vivre ensemble sur Terre. En illustrant le propos par l'exemple concret de changements récents apportés au programme de formation de l'école québécoise, notamment au sein d'un programme de Science et technologie, cet article montre comment divers éléments d'ERE peuvent contribuer à enrichir le sens de leur vie chez les élèves et ainsi construire leurs identités personnelles et sociales. On soutient également que l'intégration d'éléments d'ERE constitue une perspective pouvant contribuer à favoriser la résolution de problèmes socioécologiques et apporter un éclairage particulier à l'action citoyenne. L'article souligne également que des éléments d'ERE intégrés aux cours de sciences peuvent amener les élèves, citoyens, à participer aux affaires de leur société et à contribuer ainsi à influencer les politiques publiques. Enfin, certaines limites à une telle intégration sont présentées.

Tous droits réservés @ Association canadienne d’éducation de langue française, Ce document est protégé par la loi sur le droit d’auteur. L’utilisation des 2009 services d'Érudit (y compris la reproduction) est assujettie à sa politique d'utilisation que vous pouvez consulter en ligne.

https://apropos.erudit.org/fr/usagers/politique-dutilisation/ 


\section{L'éducation relative à l'environnement en enseignement des sciences et de la technologie : une contribution pour mieux Vivre ensemble sur Terre}

\section{Patrick CHARLAND}

Université du Québec à Montréal, Québec, Canada

\section{Patrice POTVIN}

Université du Québec à Montréal, Québec, Canada

\section{Martin RIOPEL}

Université du Québec à Montréal, Québec, Canada

\section{RÉSUMÉ}

Dans les années soixante, les sociétés du monde entier ont graduellement pris conscience de l'ampleur, de la sévérité et de la complexité des divers problèmes environnementaux qui touchent notre planète. À travers de multiples sommets, congrès et colloques, qui ont donné lieu à de nombreuses déclarations, chartes ou stratégies, le champ de l'éducation relative à l'environnement s'est graduellement constitué. Malgré des intentions et des engagements fermes exprimés par les décideurs politiques et curriculaires, il semble que les initiatives en éducation relative à l'environnement 
(ERE) se soient plus développées dans les milieux éducatifs non formels ou en contexte informel qu'en milieu scolaire. On constate cependant une tendance récente à l'intégration d'éléments d'éducation relative à l'environnement dans les curriculums, et particulièrement dans les programmes de sciences du Québec. C'est donc dans cette perspective de l'intégration de l'ERE au sein des programmes de sciences que sera traitée la question du Vivre ensemble sur Terre. En illustrant le propos par l'exemple concret de changements récents apportés au programme de formation de l'école québécoise, notamment au sein d'un programme de Science et technologie, cet article montre comment divers éléments d'ERE peuvent contribuer à enrichir le sens de leur vie chez les élèves et ainsi construire leurs identités personnelles et sociales. On soutient également que l'intégration d'éléments d'ERE constitue une perspective pouvant contribuer à favoriser la résolution de problèmes socioécologiques et apporter un éclairage particulier à l'action citoyenne. L'article souligne également que des éléments d'ERE intégrés aux cours de sciences peuvent amener les élèves, citoyens, à participer aux affaires de leur société et à contribuer ainsi à influencer les politiques publiques. Enfin, certaines limites à une telle intégration sont présentées.

\section{ABSTRACT}

\section{Environmental education in science and technology classes contribute to better "sharing the planet"}

Patrick CHARLAND

University of Quebec in Montreal, Québec, Canada

Patrice POTVIN

University of Quebec in Montreal, Québec, Canada

Martin RIOPEL

University of Quebec in Montreal, Québec, Canada

In the 1960s, societies around the world gradually became aware of the scope, severity and complexity of the diverse environmental problems affecting our planet. Despite the intentions and commitments expressed by our political and curricular decision-makers, environmental education (EE) actions seem to take place in informal educational settings, but rarely at the school. However, we notice a tendency to include elements of environmental education in the classroom, particularly as part of Québec's science programs. The question of "sharing the planet" will therefore be examined from this perspective. Illustrating the topic with the example of recent changes to the Science and Technology Program in Québec schools, this article shows how a variety of EE elements can contribute to enriching the meaning of students' lives, thus helping them to build their personal and social identities. The article also 
demonstrates that integrating EE elements can contribute to resolving socio-ecological problems, with a focus on citizen action. Certain limits to this integration approach are also presented.

\section{RESUMEN}

\section{La educación relativa al entorno ecológico en la enseñanza de ciencias y tecnología: una contribución para mejor "Vivir juntos sobre la Tierra»}

Patrick CHARLAND

Universidad de Quebec en Montreal, Quebec, Canadá

Patrice POTVIN

Universidad de Quebec en Montreal, Quebec, Canadá

Martin RIOPEL

Universidad de Quebec en Montreal, Quebec, Canadá

Durante los años sesenta, las sociedades del mundo entero tomaron gradualmente conciencia de la amplitud, la severidad y la complejidad de los diversos problemas ambientales que afectan a nuestro planeta. A pesar de las intenciones y de los compromisos expresados por los decidores políticos y curriculares, parece que las acciones en educación relativa al entorno ecológico (ERE) se realizaron en los medios educativos informales pero muy poco en las escuelas. Se constata, sin embargo, una tendencia hacia la integración de elementos de la educación relativa al entorno ecológico en los medios escolares, sobre todo en los programas de ciencias de Quebec. Es bajo ésta perspectiva que abordaremos la cuestión de «Vivir juntos sobre la Tierra». Ilustrando el discurso con ejemplos concretos de cambios recientes del programa de estudios de Ciencia y Tecnología de la escuela quebequense, éste artículo muestra cómo diversos elementos de la ERE pueden contribuir al enriquecimiento del sentido de las vidas de los alumnos y así construir sus identidades personales y sociales. El artículo afirma asimismo que la integración de elementos de la ERE es una perspectiva que puede contribuir a la resolución de problemas socioecológicos y aportar una iluminación particular a la acción ciudadana. Por último, se presentan algunos de los límites de dicha integración. 


\section{Introduction}

Cet article aborde la question du Vivre ensemble sur Terre depuis la perspective de la récente institutionnalisation scolaire de l'éducation relative à l'environnement. Particulièrement, ce sont les enjeux associés à l'intégration d'éléments d'ERE dans les programmes d'enseignement scientifique et technologique qui seront discutés. Après une présentation de certaines tendances récentes en éducation relative à l'environnement et en éducation scientifique, les nouveaux programmes québécois de Science et technologie seront succinctement décrits à titre d'exemple pour illustrer la contribution possible de tels programmes à la problématique du Vivre ensemble

L'intégration d'éléments d'éducation relative à l'environnement dans les programmes d'enseignement scientifique et technologique contribue à l'enrichissement du sens de la vie des élèves en matière de construction de leur identité environnementale. sur Terre. Premièrement, l'intégration d'éléments d'éducation relative à l'environnement dans les programmes d'enseignement scientifique et technologique contribue à l'enrichissement du sens de la vie des élèves en matière de construction de leur identité environnementale. Deuxièmement, cette intégration invite les élèves à agir pour la résolution de problèmes socioécologiques, ce qui favorise l'innovation sociale. Troisièmement, ces programmes intégrés contribuent à influencer les politiques publiques en encourageant la participation active des élèves aux affaires sociopolitiques. Enfin, seront discutés certains défis et certaines limites associés à l'intégration d'éléments d'ERE en enseignement scientifique et technologique.

\section{L'éducation relative à l'environnement à l'école}

Depuis les années soixante, les sociétés du monde entier ont graduellement pris conscience de l'ampleur, de la sévérité et de la complexité des divers problèmes environnementaux qui touchent notre planète. Cette prise de conscience s'est effectuée d'abord autour d'une multitude de problématiques d'ordre biophysique. Par exemple, Fien (1995) cite les changements climatiques, le réchauffement de la planète, la destruction de la forêt amazonienne, la décroissance de la biodiversité, la désertification de certains territoires, le déséquilibre entre les populations et les ressources disponibles, la pollution, les accidents nucléaires, l'enfouissement des déchets toxiques et plusieurs autres menaces pour la qualité de vie des humains ou l'équilibre des écosystèmes. Cet auteur souligne aussi une plus récente prise de conscience à l'égard de problématiques environnementales touchant les relations entre les individus et les groupes sociaux. En effet, les sociétés du monde entier sont progressivement, à divers degrés, conscientisées au sujet des inégalités sociales en ce qui concerne les standards de vie et de bien-être. Conflits régionaux, déséquilibres de consommation et surexploitation des ressources, famines, malnutrition, augmentation de la marginalisation des femmes et des minorités ethniques et accumulation des dettes des pays pauvres peuvent être considérés comme autant d'exemples de phénomènes sociaux associés à des problématiques environnementales.

Pour tenter de résoudre ces diverses problématiques, les acteurs des différentes organisations de gouvernance et de la société civile (chefs d'État, organismes non gouvernementaux, groupes environnementaux, syndicats, etc.) se sont rencontrés à 
l'occasion de nombreux sommets, congrès et colloques (Orellana et Fauteux, 2000). Depuis près de 50 ans, ces rencontres ont donné lieu à des engagements divers à travers de multiples rapports, chartes ou grandes déclarations ${ }^{1}$ qui témoignent de la volonté de différents décideurs et acteurs sociaux de réagir face aux problèmes environnementaux. L'éducation, tant dans les milieux formels que non formels, est vue par toutes les nations comme l'une des voies qui mèneront à une amélioration environnementale substantielle de la planète.

Malgré des intentions et des engagements fermes exprimés par les décideurs politiques et curriculaires, il semble cependant que trop peu d'actions concrètes en éducation relative à l'environnement aient été réalisées à l'école. Sauvé, Berryman et Brunelle (2003) affirment que, depuis la prise de conscience des problèmes environnementaux, l'éducation relative à l'environnement s'est surtout réalisée en contexte informel et non formel. Depuis la fin des années soixante, la plupart des initiatives en ERE provenaient des groupes écologistes, des associations d'éducation populaire, des médias, etc.

Dans une recherche récente, Sauvé, Berryman et Brunelle (2003) ont examiné l'état de l'institutionnalisation scolaire de l'éducation relative à l'environnement dans le monde. Ils constatent une tendance récente à l'intégration d'une dimension environnementale à l'école, en particulier à la faveur de l'engouement pour le «développement durable». D'abord, ils remarquent que l'intégration d'éléments d'ERE s'opérationnalise selon divers scénarios. L'éducation relative à l'environnement est surtout introduite par la stratégie de la transversalité, où l'environnement est présenté comme un thème transversal, un lieu d'intégration pour diverses disciplines scolaires. C'est le cas de plusieurs curriculums réformés, par exemple ceux des États-Unis, du Canada (Conseil des ministres de l'Éducation du Canada, 1997), du Québec (Gouvernement du Québec, 1997), de la Grande-Bretagne (Gayford et Dillon, 1995) et de l'Espagne (Membiela, 1999).

D'autres scénarios curriculaires, comme c'est le cas en Grèce, proposent une intégration de l'ERE à travers certains projets transdisciplinaires relatifs à des problématiques environnementales spécifiques. Papadimitriou (2001) rapporte que ces «modules » sont souvent annexés de manière plutôt accessoire au curriculum officiel. Dans une analyse générale portée au début des années 2000, elle signalait qu'en dépit de l'enthousiasme à propos de l'éducation relative à l'environnement à l'école son arrimage avec les disciplines scolaires était problématique:

Plusieurs progrès ont été faits par la production de matériel pédagogique, de haute qualité dans plusieurs cas, plusieurs pratiques fructueuses ont été rapportées, mais, concrètement, l'éducation relative à l'environnement n'a pas été un sujet abordé par tous les élèves. Aucun pays ne peut aujourd'hui se réclamer d'avoir complètement intégré l'éducation relative à l'environnement

1. À titre d'exemple, on peut citer la Déclaration de Stockholm (1972), la Charte de Belgrade (1975), la Déclaration de Tbilissi (1977), le Rapport Brundtland (1987), la Déclaration de Rio (1992), la Déclaration de Johannesburg sur le développement durable (2002). 
dans son curriculum scolaire, et ce, malgré les multiples affirmations officielles en ce sens (Traduction libre, Papadimitriou, 2001).

Jusqu'à récemment, il semble que peu de gouvernements avaient concrètement introduit l'ERE dans leurs curriculums scolaires sous forme d'objets d'apprentissage spécifiques ou de programmes distincts. Pendant des dizaines d'années, de nombreux curriculums nationaux ont fait référence à l'ERE de façon générale dans leurs principes et fondements éducatifs (Sauvé, 1997), mais sans que cela ait de véritables répercussions dans les pratiques d'enseignement/apprentissage (Papadimitriou, 2001).

En ce qui concerne les disciplines concernées par l'intégration d'éléments d'ERE, Sauvé, Berryman et Brunelle (2003) mentionnent que les créneaux les plus fréquents sont ceux des sciences biophysiques, des sciences humaines et du développement personnel et social. Cela dit, plusieurs auteurs (Andrew et Robottom, 2001; Ashley, 2000; Korfiatis, 2005; Sauvé, 1997) défendent l'idée que c'est surtout dans les disciplines scientifiques que l'éducation relative à l'environnement tend à s'intégrer en contexte scolaire.

\section{Un changement de perspective en éducation scientifique}

L'intégration des préoccupations environnementales en enseignement des sciences concorde avec un vent de changement en éducation scientifique. Depuis le début des années quatre-vingt-dix, plusieurs spécialistes (Solomon, 1988; Fourez,

Plusieurs études soulignent que les modèles traditionnels d'enseignement des sciences, basés sur la transmission de contenus, ont notamment eu pour effet d'entraîner chez les élèves une importante baisse de motivation et d'intérêt face aux questions et aux carrières scientifiques. 1994; Giordan et al., 1989) et organisations œuvrant en éducation (en particulier l'Unesco, 1985; American Association for the Advancement of Science, 1993) ont fait le constat que l'éducation scientifique en général et plus spécifiquement l'enseignement des sciences étaient en état de crise. Dans les anciens programmes basés sur un modèle monodisciplinaire de l'enseignement des sciences, on constatait que les élèves étaient rarement en mesure de transférer les connaissances scientifiques apprises dans un cadre scolaire à des situations nouvelles ou à leur quotidien (Östman, 1998). Plusieurs études (Organisation de coopération et de développement économiques, 1996; Convert et Gugenheim, 2005) soulignent que les modèles traditionnels d'enseignement des sciences, basés sur la transmission de contenus, ont notamment eu pour effet d'entraîner chez les élèves une importante baisse de motivation et d'intérêt face aux questions et aux carrières scientifiques.

Pour Fourez (1998), le cloisonnement disciplinaire caractéristique de la pédagogie par objectifs a certainement amené de l'ordre et de l'organisation dans les curriculums, mais aussi une perte de sens. Selon Beane (1997), enseigner les sciences dans une approche disciplinaire correspond aujourd'hui à une fin en soi et non à un moyen qui peut être utilisé pour résoudre des problèmes liés à la réalité du jeune dans sa vie quotidienne. Fourez, Maingain et Dufour (2002, p. 28) abondent dans ce sens en soulignant que « seule une nouvelle organisation des curriculums, mettant en réseau les savoirs et les compétences des différents champs disciplinaires, est susceptible de répondre aux exigences actuelles de nos sociétés ». 
D'une ancienne perspective où l'on visait la formation pointue de futurs scientifiques, les nouveaux programmes intégrés visent le développement d'une culture scientifique chez l'élève. L’idée est de préparer adéquatement les élèves citoyens de manière à ce qu'ils puissent comprendre les enjeux de leur société et y jouer un rôle actif.
Ainsi, l'une des solutions à cette crise de l'enseignement des sciences résiderait dans l'idée générale d'interdisciplinarité curriculaire (Lenoir et Sauvé, 1998; Fourez, 1994) ou de curriculum intégrateur (Beane, 1997). Ce type de curriculum implique souvent un enseignement et un apprentissage organisés autour de situations (Jonnaert, 2006), de questions, de problématiques ou de phénomène signifiants (Östman, 1998), plutôt qu'en fonction de la traditionnelle structure des disciplines scientifiques.

Cette réorganisation des curriculums est cependant lourde de conséquences. D'une ancienne perspective où l'on visait la formation pointue de futurs scientifiques, les nouveaux programmes intégrés visent le développement d'une culture scientifique chez l'élève. L'idée est de préparer adéquatement les élèves citoyens de manière à ce qu'ils puissent comprendre les enjeux de leur société et y jouer un rôle actif.

La perspective du développement d'une culture scientifique constitue une tendance lourde dans de nombreux programmes scientifiques réformés. Au Québec, cette perspective prend forme par l'intégration des disciplines scientifiques entre elles, de même que par l'intégration de nouveaux champs d'intervention éducative comme l'éducation technologique ou l'éducation relative à l'environnement.

\section{Un exemple innovateur d'intégration de l'ERE à l'école: les nouveaux programmes de sciences et de technologie du Québec}

Au Québec, la tendance à l'intégration d'éléments d'éducation relative à l'environnement est présente, d'une manière considérée comme innovante, à travers une réforme du curriculum en général et, particulièrement, dans les nouveaux programmes en enseignement des sciences. Décrite dans le Programme de formation de l'école québécoise (PFEQ), cette réforme est maintenant implantée au primaire et dans presque tout le secondaire (Gouvernement du Québec, 2003). Le PFEQ adopte généralement «la perspective de connaissances construites par l'élève plutôt que transmises par l'enseignant [...]» (Gouvernement du Québec, 2003, p. 9). Alors que l'enseignant avait traditionnellement un rôle de transmetteur de connaissances, ce nouveau curriculum vise le développement de compétences dans le cadre de situations d'apprentissage contextualisées et abordant des réalités signifiantes.

Pour opérationnaliser cette contextualisation des situations d'apprentissage, le ministère de l'Éducation a introduit l'idée de domaines généraux de formation (DGF), qui représentent diverses "problématiques auxquelles les jeunes doivent faire face dans différentes sphères importantes de leur vie " (Gouvernement du Québec, 2003, p. 21) et qui sont porteuses d'enjeux majeurs pour les individus et les collectivités. Les DGF, au nombre de cinq, sont regroupés de la manière suivante : «santé et bienêtre », «orientation et entrepreneuriat ", «vivre-ensemble et citoyenneté», «médias » et « environnement et consommation». 


\section{Intégration transversale de I'ERE à toutes les disciplines du Programme de formation de l'école québécoise}

C'est entre autres par le domaine général de formation « environnement et consommation" que certains éléments d'éducation relative à l'environnement sont introduits dans tous les programmes disciplinaires du PFEQ, dont ceux du profil scientifique. Ainsi, le gouvernement du Québec (2003, p. 25) souligne que «dans le domaine de l'environnement, l'école doit amener les jeunes à appréhender les rapports de l'homme à l'univers [...], en prenant davantage conscience de l'interdépendance des systèmes à l'échelle planétaire». En contextualisant des situations d'apprentissage par enjeux environnementaux, le PFEQ semble vouloir conscientiser les élèves à l'égard de l'influence que peuvent avoir leurs propres actions sur la préservation d'un milieu dont leurs conditions de vie sont largement tributaires. Cela pourra les sensibiliser « aux effets à long terme de l'utilisation incontrôlée ou de l'exploitation abusive des ressources naturelles» (Gouvernement du Québec, 2003, p. 25).

Intégrés de manière transversale à toutes les disciplines, les enjeux environnementaux, ainsi que le souligne le PFEQ (2003, p. 26), «apportent des éclairages complémentaires sur les dimensions sociale, politique, économique, scientifique, technologique et éthique qui marquent les rapports de l'homme à son environnement». Ces enjeux permettent donc de nourrir la réflexion sur les multiples facteurs qui façonnent les habitudes de vie des humains, notamment en matière de consommation, et sur leurs conséquences à l'échelle de la planète. Cette réflexion vise à permettre à l'élève d'inférer les conséquences de ses actes sur son milieu et d'adopter ainsi un comportement responsable à l'égard de son environnement.

\section{Intégration spécifique en science et technologie}

En lien avec la voie de la transversalité (Sauvé, Berryman et Brunelle, 2003) ou celle des projets transdisciplinaires (Papadimitriou, 2001), le Programme de formation de l'école québécoise intègre des éléments d'ERE de manière particulièrement innovante et inédite à travers les nouveaux programmes de Science et technologie. Ces programmes mettent en relation plusieurs champs disciplinaires (sciences de la Terre et de l'espace, biologie, physique, chimie, technologie, etc.). Plutôt que de viser la formation de futurs scientifiques, souvent orientée vers les connaissances à apprendre, ces programmes misent sur le développement de compétences disciplinaires pour développer une culture scientifique et technologique qui permettra aux élèves de mieux comprendre les enjeux socioscientifiques de leur société.

À travers les programmes de science et technologie du secondaire, l'intégration d'éléments d'éducation relative à l'environnement prend des formes diverses selon les niveaux scolaires. Au premier cycle du secondaire, l'élève est amené à appréhender son environnement par la compréhension de phénomènes naturels. Aussi, l'élève abordera des questions environnementales par une évaluation des retombées des sciences et de la technologie. Cette façon d'intégrer des éléments d'ERE n'est pas nouvelle. Elle rappelle la perspective Science-Technologie-Société (STS) (Aikenhead, 1987), proposée à la fin des années quatre-vingt au Canada anglais et aux États-Unis, ou celle de Science-Technologie-Environnement-Société (STES) formalisée en Israël (Zoller, 1992). 
Ce qui confère toutefois au curriculum québécois un caractère véritablement novateur concerne le programme ordinaire de $4^{\mathrm{e}}$ secondaire, entièrement organisé autour de problématiques environnementales : les changements climatiques, la déforestation, I'eau et l'énergie. II est à ce jour l'un des seuls programmes d'État à être entièrement orientés et structurés de cette manière.
Ce qui confère toutefois au curriculum québécois un caractère véritablement novateur concerne le programme ordinaire de $4^{\mathrm{e}}$ secondaire, entièrement organisé autour de problématiques environnementales: les changements climatiques, la déforestation, l'eau et l'énergie. Il est à ce jour l'un des seuls programmes d'État à être entièrement orientés et structurés de cette manière. Tous les concepts constituant le contenu de ce programme obligatoire de $4^{\mathrm{e}}$ secondaire ont été systématiquement sélectionnés en fonction de leur pertinence pour la compréhension des problématiques à l'étude.

Les élèves construisent des savoirs et développent des compétences en science et technologie en abordant des problématiques environnementales et en développant simultanément des compétences relatives à l'environnement. Ainsi, les élèves sont amenés à construire leur opinion sur différents aspects des problématiques abordées. Pour les élèves qui se destinent à une carrière scientifique, deux autres programmes optionnels - mais complémentaires au programme ordinaire -, ont été élaborés selon les mêmes modalités: les programmes Science et technologie de l'environnement ( $4^{\mathrm{e}}$ secondaire) et Science et environnement ( $4^{\mathrm{e}}$ secondaire) sont organisés autour deux autres problématiques environnementales : l'alimentation et les matières résiduelles.

Il est pertinent de souligner que cette tendance à l'intégration de préoccupations environnementales n'est pas exclusive à certains programmes à caractère scientifique et technologique. En effet, les programmes Éthique et culture religieuse, Éducation à la citoyenneté ou Géographie prévoient, selon leurs propres perspectives disciplinaires, l'étude d'enjeux environnementaux. Cela dit, les programmes de science et technologie de $4^{\mathrm{e}}$ secondaire se distinguent par le fait que les problématiques environnementales sont au cœur même de leurs structures et de leurs objectifs d'apprentissage.

\section{L'intégration d'éléments d'ERE en enseignement des sciences et de la technologie : une contribution au mieux-vivre ensemble sur terre}

Ayant jusqu'ici traité des modalités d'intégration curriculaire de l'ERE en éducation scientifique et plus particulièrement en enseignement des sciences, nous visons dans la section suivante à montrer en quoi une telle intégration contribue à la question du mieux-Vivre ensemble sur Terre, fil conducteur de ce numéro thématique. Cette section montre ainsi de manière précise comment un programme, comme celui de Science et technologie de $4^{\mathrm{e}}$ secondaire au Québec, permettra, à sa manière, d'enrichir le sens de la vie des élèves, de contribuer à l'innovation sociale et d'influencer les politiques publiques. 


\section{L'ERE en enseignement de la science et de la technologie : enrichir le sens de la vie des élèves}

Dans une perspective fondamentale, l'intégration d'éléments d'éducation relative à l'environnement permet d'aborder la question du développement personnel et social de manière renouvelée. À travers les systèmes et les institutions scolaires, Legendre (2005) précise que l'éducation a notamment pour finalité le développement des individus (apprenants) eux-mêmes, en relation avec les autres (apprenants, adultes, acteurs de la communauté, etc.). Il semble cependant que la question du rapport à l'environnement, du rapport à cet Oïkos, la maison de vie partagée par les humains (Sauvé, 2001), ait été relativement absente des curriculums jusqu'aux mouvements récents des systèmes éducatifs. Or, comme le rappelle Tilbury (1994), il est important que les enfants soient amenés à développer le plus tôt possible leur rapport avec l'environnement.

Plusieurs des nouveaux programmes de sciences, dont ceux du Québec, sont réformés dans la perspective de rendre les apprentissages plus signifiants. La ruelle, le boisé du quartier, son chez-soi, la cour d'école sont autant de lieux qui permettent de contextualiser et de rendre signifiants divers apprentissages en sciences et technologie. Contextualiser l'étude de phénomènes naturels ou impliquer les élèves dans la résolution de diverses problématiques environnementales, globales ou locales, peut donc stimuler l'apprentissage scolaire, tout en développant chez l'apprenant une identité personnelle, sociale et environnementale.

Néanmoins, il est pertinent d'ajouter, comme le rappelle Sauvé (2001), que l'éducation relative à l'environnement à l'école ne doit pas seulement se réduire à une thématique environnementale qui permet de contextualiser les apprentissages d'une discipline particulière. L'éducation relative à l'environnement constitue en soi un projet de développement humain, qui serait alors à intégrer à toutes les disciplines scolaires, dont les sciences.

\section{L'ERE en enseignement de la science et la technologie : contribuer à l'innovation sociale}

Selon Klein et Harrisson (2006, p. 389), l'innovation sociale « peut être envisagée comme un processus d'apprentissage dont l'objectif consiste en la valorisation individuelle et l'autonomisation des acteurs en vue de leur permettre de résoudre leurs problèmes ». Ces auteurs ajoutent que le concept d'innovation sociale peut également être abordé par la notion de mieux-être, des individus ou des collectivités.

C'est dans cette perspective que l'intégration d'éléments d'éducation relative à l'environnement en enseignement des sciences et technologie peut contribuer à favoriser l'innovation sociale et qu'elle permet d'apporter un éclairage particulier à l'action citoyenne. Comme le soutiennent Ashley (2000) et Gayford (2002), les programmes de sciences constituent de plus en plus un point de départ dans le développement de curriculums nationaux qui pourront avoir une influence dans la résolution des problématiques socioenvironnementales locales, régionales ou même globales.

Les enseignants de sciences ont non seulement pour rôle de transmettre des connaissances ou de développer des compétences de résolution de problème, mais 
Les enseignants de sciences ont non seulement pour rôle de transmettre des connaissances ou de développer des compétences de résolution de problème, mais ils ont dorénavant la responsabilité de former des élèves conscientisés et critiques à l'égard des impacts que peuvent avoir les sciences et technologies sur la nature et sur les espèces qui y vivent. ils ont dorénavant la responsabilité de former des élèves conscientisés et critiques à l'égard des impacts que peuvent avoir les sciences et technologies sur la nature et sur les espèces qui y vivent. Sauvé (2006) mentionne qu'une telle visée avait été, jusqu'à récemment, peu soutenue par les organisations scolaires. Or, la mouvance des changements éducationnels récents permet d'« espérer que les propositions des programmes de formation soient de nature à favoriser un virage pédagogique, entre autres à travers un enseignement en science et technologie axé sur l'investigation critique, l'heuristique et la pertinence sociale» (Sauvé, 2006, p. 8).

\section{L'ERE en enseignement de la science et la technologie : influencer les politiques publiques}

Enfin, des éléments d'ERE à travers l'enseignement scientifique et technologique peuvent contribuer à influencer les politiques publiques en amenant les élèves, écocitoyens, à participer aux affaires publiques de leur société. En effet, la compréhension et l'action des élèves à l'égard des problématiques environnementales locales ou globales sont de nature à favoriser leur participation aux débats sociaux, et donc à influencer les politiques publiques.

Selon Désautels (1998), les modèles traditionnels d'enseignement des sciences favorisaient une attitude passive de la part des élèves dans leurs rôles de citoyens. Étant donné que la science et la technologie leur étaient présentées comme étant l'affaire d'experts, ces derniers étaient souvent considérés comme étant les seuls, donc les mieux placés, pour intervenir dans ces domaines. Ainsi, les élèves avaient souvent tendance à s'exclure des débats socioscientifiques qui les concernent directement. Dans une approche sociopolitique de l'enseignement, il s'agit pour l'enseignant de mettre ses élèves en situations réelles d'engagement social. Selon Désautels (1998), les élèves peuvent, à travers cet engagement, acquérir des connaissances et développer des compétences scientifiques en contexte; ils peuvent par ailleurs mieux comprendre la nature des enjeux socioenvironnementaux. Un programme comme celui de Science et technologie de $4^{\mathrm{e}}$ secondaire constitue une porte toute grande ouverte vers ce genre d'engagement social qui permettrait certainement de stimuler la participation citoyenne des élèves.

\section{Des défis et limites à l'intégration de l'ERE dans l'enseignement des sciences}

Pour certains décideurs curriculaires, enseignants ou spécialistes de la didactique des sciences, l'intégration de l'éducation relative à l'environnement aux programmes d'enseignement scientifique et technologique constitue une avenue prometteuse mais limitée.

Tout d'abord, Sauvé (1997) rapporte que plusieurs enseignants voient en cette intégration une menace d'appauvrissement des contenus scientifiques, qui doivent être retirés ou dilués au profit de l'ERE. Aussi, Thésée (2008) soutient que l'ERE confiée aux enseignants de sciences et technologie constitue un paradoxe de transposition 
didactique. Elle met en lumière diverses incohérences ontologiques, axiologiques et épistémologiques liées à la conjugaison de l'enseignement des sciences et de la technologie avec l'éducation relative à l'environnement.

Dans une perspective semblable, Charland (2006-2007) a étudié les interfaces entre les champs d'intervention éducative de l'enseignement des sciences, de l'enseignement de la technologie et l'éducation relative à l'environnement (ERE). Il cite de nombreux auteurs qui relèvent le même genre d'incohérence. L'éducation relative à l'environnement, par sa visée d'optimalisation du rapport à l'environnement, favorise le développement d'une éthique de liens avec l'environnement qui est axée sur des valeurs comme le respect, la responsabilité, la solidarité ou l'engagement. Cette perspective est fort différente de celle d'un enseignement des sciences basé sur la compréhension «objective» du monde et guidé par valeurs de rationalité, de rigueur ou de reproductibilité. Selon Andrew et Robottom (2001), l'intégration cohérente entre l'ERE et l'enseignement scientifique ne sera possible que lorsque les idées ou croyances positivistes à l'égard de l'activité scientifique ne seront plus dominantes chez les enseignants de sciences.

En plus de ces divergences théoriques, certains auteurs soulèvent des enjeux pratiques à cette mise en relation. Sauvé (1997) met ainsi en évidence plusieurs facteurs limitants dans l'intégration de l'ERE à l'école québécoise : surcharge des programmes, condition enseignante, manque de formation, manque de matériel et saturation des enseignants ou des élèves à l'égard des préoccupations environnementales. Pour sa part, Korfiatis (2005) rapporte que plusieurs spécialistes ont remis en question, pour des raisons pratiques, les disciplines scientifiques scolaires comme lieu d'intégration à privilégier pour l'ERE. Ces auteurs soutiennent que les enseignants de sciences peuvent certainement faire référence à des concepts liés à l'environnement, mais ils doutent de la compétence réelle des enseignants de sciences à inclure des dimensions éducatives liées à l'environnement. L'ERE invite les enseignants et les élèves à participer, de diverses manières, à des débats démocratiques prenant en compte les aspects moraux, éthiques et politiques des questions socioécologiques, et cela interpelle l'univers des valeurs et attitudes individuelles et sociales. Or, considérant que ces dimensions ne font pas intrinsèquement partie de leurs cours, de nombreux enseignants de sciences (Sauvé, 1997; Ashley, 2000) ou de technologie (Dillon, 2000) évitent volontairement de les aborder avec leurs élèves. Sauvé (2006) rappelle que cette réaction est normale dans un contexte où les enseignants n'ont été que très peu formés à l'égard des questions environnementales et des enjeux d'éducation relative à l'environnement.

\section{Conclusion}

Malgré les défis et les limites soulevés, nous avons tenté de souligner dans ce texte la contribution possible de l'intégration de l'ERE à l'enseignement des sciences et technologie au mieux-Vivre ensemble sur Terre. En vue de répondre aux arguments d'incohérences épistémologiques entre l'ERE et l'enseignement des sciences, nous 
Les enseignants ont maintenant la possibilité de rendre signifiants et intéressants les savoirs et les compétences développés dans leurs cours, tout en contribuant au développement de l'identité environnementale de leurs élèves, à la résolution des problèmes socioécologiques ainsi qu'à la participation de leurs élèves aux affaires publiques. sommes d'avis, à l'instar de Bader (1998), qu'il est primordial que s'amorce un renversement épistémologique de manière à assouplir les visions souvent trop positivistes ou scientistes des enseignants de sciences. Or, les nouvelles visées des programmes réformés, qui sont orientés vers le développement d'une culture en science et technologie favorisant la formation de citoyens critiques, semblent justement être à la fois le reflet et l'amorce majeure d'un tel renversement épistémologique.

Bien que la perspective scientifique ou technologique ne soit pas la seule à privilégier quant à l'intégration scolaire de l'ERE, il n'en demeure pas moins que les savoirs ou compétences scientifiques/technologiques sont des éléments incontournables dans la compréhension des phénomènes et des problématiques de nature environnementale. Cette compréhension constitue un premier pas vers une éducation visant à favoriser de meilleurs rapports avec l'environnement.

Dans le contexte actuel de changements éducationnels, il importe à mon sens de former, mais surtout de sensibiliser les enseignants de sciences au rôle prépondérant qu'ils sont appelés à jouer dans cette question du mieux-Vivre ensemble sur Terre. Les enseignants ont maintenant la possibilité de rendre signifiants et intéressants les savoirs et les compétences développés dans leurs cours, tout en contribuant au développement de l'identité environnementale de leurs élèves, à la résolution des problèmes socioécologiques ainsi qu'à la participation de leurs élèves aux affaires publiques.

\section{Références bibliographiques}

AIKENHEAD, G.S. (1987). Views on Science-Technology-Society. Saskatchewan : Department of Curriculum Studies, University of Saskatchewan.

AMERICAN ASSOCIATION FOR THE ADVANCEMENT OF SCIENCE (1993). Science for All American, Project 2061. New York : Oxford University Press.

ANDREW, J. et ROBOTTOM, I. (2001). Science and Ethics: Some Issues for Education. Science Education, 85, 769-780.

ASHLEY, M. (2000). Science: An Unreliable Friend to Environmental Education? Environmental Education Research, 6, 269-280.

BADER, B. (1998). Une vision socialisée des sciences au service d'une éducation relative à l'environment socioconstructiviste. Canadian Journal of Environmental Education, 3, 156-170.

BEANE, J.A. (1997). Curriculum Integration: Designing the Core for Democratic Education. New York: Teachers College Press. 
CHARLAND, P. (2006-2007). La triade science, technologie et environnement: nouveaux enjeux théoriques, curriculaires et pédagogiques. Éducation relative à l'environnement: Regards - Recherches - Réflexions, 6, 61-72.

CONSEIL DES MINISTRES DE L'ÉDUCATION DU CANADA (1997). Cadre commun de résultats d'apprentissage en sciences de la nature. Toronto : Gouvernement du Canada.

CONVERT, B. et GUGENHEIM, F. (2005). Scientific Vocations in Crisis in France: Explanatory Social Developments and Mechanisms. European Journal of Education, 40.

DÉSAUTELS, J. (1998). Une éducation aux technosciences pour l'action sociale. La recherche en didactique au service de l'enseignement. Marrakech, Maroc: Université Cadi Ayyad.

DILLON, J. (2000). Perspectives on Environmental Education-Related Research in Science Education. Relations between Science Education and Environmental (Science) Education. A NARST Symposium. 27 mars 2001.

FIEN, J. (1995). Teaching for a Sustainable World: The Environmental and Development Education Project for Teacher Education. Environmental Education Research, 1, 21-33.

FOUREZ, G. (1994). Alphabétisation scientifique et technique: essai sur les finalités de l'enseignement des sciences. Bruxelles : De Boeck Université.

FOUREZ, G. (1998). Se représenter et mettre en œuvre l'interdisciplinarité à l'école. Revue des sciences de l'éducation, 24, 31-50.

GAYFORD, C. et DILLON, P.J. (1995). Policy and the Practice of Environmental Education in England: A Dilemma for Teachers. Environmental Education Research, 1, 173-183.

GIORDAN, A., HENRIQUES, A. et BANG, V. (1989). Psychologie génétique et didactique des sciences. Berne : P. Lang.

GOUVERNEMENT DU QUÉBEC (1997). Prendre le virage du succès : réaffirmer l'école. Québec: ministère de l'Éducation.

GOUVERNEMENT DU QUÉBEC (2003). Programme de formation de l'école québécoise. Enseignement secondaire premier cycle. Un programme de formation pour le XXI siècle. Québec: ministère de l'Éducation, du Loisir et du Sport.

JONNAERT, P. (2006). Action et compétence, situation et problématisation, dans M. Fabre et É. Vellas (dir.), Situations de formation et problématisation (p. 31-40). Genève: De Boeck Université.

KLEIN, J.-L. et HARRISSON, D. (2006). L'innovation sociale. Québec : Presses de l'Université du Québec. 
KORFIATIS, K.J. (2005). Environmental Education and the Science of Ecology: Exploration of an Uneasy Relationship. Environmental Education Research, 11, 235-248.

LENOIR, Y. et SAUVÉ, L. (1998). De l'interdisciplinarité scolaire à l'interdisciplinarité dans la formation à l'enseignement: un état de la question. Revue française de pédagogie, 124, 127-143.

MEMBIELA, P. (1999). Towards the Reform of Science Teaching in Spain: the Social and Personal Relevance of Junior Secondary School Science Projects for a Socially Responsible Understanding of Science. International Journal of Science Education, 21, 721-730.

ORELLANA, I. et FAUTEUX, S. (2000). L'éducation relative à l'environnement à travers les grands moments de son histoire, dans A. Jarnet, B. Jickling, L. Sauvé, A. Wals et P. Clarkin (dir.), Proceedings from an On-Line Colloquium. The Future of Environnemental in a Post-modern World? (p. 13-24). Whitehorse : Yukon College.

ORGANISATION DE COOPÉRATION ET DE DÉVELOPPEMENT ÉCONOMIQUES (1996). Changing the Subject, dans P. Black et J.M. Atkin (dir.), Innovations in Science, Mathematics and Technology Education. Londres : Routledge.

ÖSTMAN, L. (1998). How Companion Meanings Are Expressed by Science Education Discourse, dans D.A. Roberts et L. Östman (dir.), Problems of Meaning in Science Curriculum (p. 54-70). New York: Teachers College Press.

SAUVÉ, L. (1997). L'éducation relative à l'environnement à l'école secondaire québécoise : état de la situation. Rapport d'une enquête diagnostique dans le cadre d'un programme de recherches intitulé Théories et pratiques de l'éducation relative à l'environnement à l'école secondaire québécoise. Montréal : Université du Québec à Montréal, Centre interdisciplinaire de recherches sur l'apprentissage et le développement en éducation.

SAUVÉ, L. (2001). L'éducation relative à l'environnement. Une dimension essentielle de l'éducation fondamentale, dans C. Goyer et S. Laurin (dir.), Entre culture, compétence et contenu: la formation fondamentale, un espace à redéfinir (p. 293-318). Montréal: Éditions Logiques.

SAUVÉ, L. (2006). Environnement et consommation : stimuler l'engagement et construire l'espoir. Spectre, 36, 8-11.

SAUVÉ, L., BERRYMAN, T. et BRUNELLE, R. (2003). Environnement et développement: la culture de la filière ONU. Éducation relative à l'environnement : Regards Recherches-Réflexions, 4, 33-55.

SOLOMON, J. (1988). The Dilemma of Science, Technology and Society Education, dans P.J. Fensham (dir.), Development and Dilemmas in Science Education (p. 266-281). New York: Falmer Press. 
THÉSÉE, G. (2008). Un paradoxe de transposition didactique : l'éducation relative à l'environnement confiée à l'enseignement des sciences et technologie. Spectre, $38,50-53$.

TILBURY, D. (1994). The Critical Learning Years for Environmental Education, dans R. Wilson (dir.), Environmental Education at the Early Childhood Level. Washington : North American Association for Environmental Education.

UNESCO (1985). Vers une pédagogie de résolution de problèmes. Paris, UNESCO.

ZOLLER, U. (1992). The Technology/Education Interface: STES Education for All. Canadian Journal of Education, 17, 86-91. 